\section{Lipides et cerveau}

Les journées Chevreul 2007 « Lipides et Cerveau » organisées conjointement par I'AFECG (Association Française de l'Etude des Corps Gras) et la DGF (Deutsche Gesellschaft für Fettwissenschaft) sous les auspices d'EuroFedLipid, ont réuni plus de 150 participants en provenance de 13 pays. Les industriels étaient bien représentés (45\% de l'auditoire), mais également des académiques (30 \%) et un certain nombre d'étudiants étrangers.

Cesymposium etlavingtaine deconférenciers qu'il a réunis, ont permis de faire le point sur les études les plus récentes concernant le rôle des acides gras polyinsaturés (AGPI), essentiellement des oméga-3 ( $n-3)$ : acide alphalinolénique (ALA : 18:3n-3) précurseur des longues chaînes (LCn-3), comme l'acide eicosapentaénoïque (EPA : 20:5n-3) et l'acide docosahexaénoïque (DHA : 22:6n-3).

Compte tenu des dimensions cognitives et comportementales et des enjeux de santé publique qui leur sont attachés, ces sujets sont devenus une part essentielle des études lipidiques :

- Quels effets positifs peuvent être attribués aujourd'hui aux oméga-3, dans les trois situations critiques de la vie humaine : en début de développement, en fin de vie et dans toutes les pathologies ou prévention des pathologies du cerveau?

- Quelles informations ou certitudes, les études épidémiologiques nous délivrentelles sur le rôle de la nutrition et des apports en lipides alimentaires spécifiques (AGPI) sur les fonctions du cerveau et le comportement humain ?

- Où en sont les études des mécanismes par lesquels ces acides gras interviennent au sein des processus de développement ou de maintien des structures et fonctions cérébrales?

Avec les éléments de réponse apportés à ces questions essentielles ont été aussi présentés les projets et moyens mis en œuvre (scientifiques et technologiques) visant à rendre disponible des sources de LCn-3 nécessaires à l'alimentation humaine, autres que les huiles de poisson.

La qualité, la diversité ainsi que la nouveauté des résultats présentés, issus d'études épidémiologiques, d'observa- tion chez I'homme et I'animal ou issus d'études plus fondamentales (culture cellulaire, distribution in vivo des acides gras après marquage...) sont à souligner.

\section{Quelques points marquants}

II est maintenant bien connu que des apports en LC pendant la gestation et les premiers mois de la vie sont essentiels pour la croissance de l'enfant et le développement du cerveau. Les LCn-6 comme l'arachidonique (AA : 20:4n-6) et les LCn-3 comme le DHA sont déposées dans les cellules neuronales et vont conditionner la structure, la fluidité et les fonctions des membranes cérébrales. Les fonctions du cerveau, tout au long de la vie, vont donc dépendre des interactions entre ces acides gras, qui sont nutritionnellement « essentiels » (indispensables), comme leurs précurseurs respectifs (linoléique n-6 et alphalinolénique $n-3$ ). Ne pouvant être synthétisés de novo, ils doivent donc être apportés par l'alimentation.

Ainsi, pendant la vie fotale, le contenu en $L C n-3$ et $n-6$ (DHA et AA) est plus élevé dans la circulation fœtale que dans la circulation maternelle, parce qu'il existe un transfert placentaire sélectif pour ces LC (via des protéines de liaison et de transfert), et plus particulièrement pour le DHA comme l'a rapporté H. Demmelmair.

Cependant, les besoins du nouveauné (première semaine) étudiés par R. Pawlosky, à partir de systèmes sophistiqués utilisant le marquage isotopique d'acides gras, montrent également qu'un apport additionnel d'AA de $4 \mathrm{mg} / \mathrm{kg} / \mathrm{j}$ serait nécessaire pour satisfaire aux besoins métaboliques cruciaux durant cette période où i) le transfert placentaire des nutriments a cessé, ii) où l'enfant perd du poids et iii) où il est obligé de mobiliser ses réserves lipidiques.

L'accumulation de DHA dans les membranes neuronales est particulièrement élevée pendant cette période, pour les besoins de la myelinisation et de la synaptogenèse.

S. Vancassel a montré qu'un défaut en DHA du cerveau de rat, lié à une carence nutritionnelle sévère en oméga-3 sur plusieurs générations, entraîne dans la descendance une hyperactivité et des défauts d'apprentissage, liés à un déficit de neurotransmission. Ce déficit peut être restauré dans certaines circonstances par modification nutritionnelle en réintroduisant des oméga-3.

Il est important de souligner que toutes ces études, pour mettre en évidence les effets du déficit en DHA cérébral, utilisent des régimes carencés en ALA par comparaison avec des régimes apportant de l'huile de colza (riches en ALA), c'est-à-dire des régimes ne comportant que le précurseur $n-3$ du DHA, sans apport alimentaire direct de DHA préformé. Dans ces conditions expérimentales, c'est le DHA issu de la transformation in vivo de I'ALA alimentaire qui fait la différence au niveau du cerveau.

L'importance des coopérations neurones/ astrocytes/cellules endothéliales a été soulignée par les mêmes auteurs, ainsi que le lien avec le métabolisme et les transporteurs cérébraux au glucose (P. Guesnet, F. Pifferi, Groupe NuRéLice). $\mathrm{Si}$ les études précitées utilisent des modèles animaux présentant des carences importantes qui ont permis d'identifier les besoins en oméga-3 pour un maintien dit «optimal » des fonctions cérébrales, chez l'homme on ne rencontre que très rarement ces cas très graves. On observe plus généralement des apports insuffisants en oméga3 associés à de simples réductions du DHA plasmatique, qui sont cependant impliqués dans des déficits d'acuité visuelle (N. Accar) et corrélés à différents troubles du cerveau liés ou non à l'âge : déclin cognitif, maladie d'Alzheimer, troubles du comportement, hyperactivité, dépression, épilepsie...

La très bonne revue de P. Astorg de toutes les études épidémiologiques réalisées dans différents pays, montre clairement l'importance de la consommation de poissons riches en LCn-3, dans la survenue ou la correction des «mood disorders", dépression, suicides. L'association est d'autant plus forte que les phénomènes sont sévères (suicides) ou récurrents pour la dépression. Une étude indique également que I'ALA plasmatique est diminué dans un petit groupe de dépressifs et qu'un traitement avec des doses modérées d'EPA peut induire un effet bénéfique (résultats encore préliminaires). 
Des études épidémiologiques rapportées par P. Barberger-Gateau sur la démence et la maladie d'Alzheimer vont dans le même sens : protection avec le degré de consommation de poisson, mais aussi avec la consommation globale d'oméga3 , incluant I'ALA. L'étude des TroisCités menée depuis plus de 4 ans par P. Barberger-Gateau, apporte les mêmes conclusions: la consommation de poisson une fois la semaine est associée à une réduction du risque, mais seulement chez les non-porteurs de l'allèle ApoE4. De plus, elle montre clairement que la consommation régulière d'huiles riches en ALA (noix/colza) est également associée à une réduction du risque, alors qu'une consommation d'huiles riches en oméga- 6 , non compensée par une consommation d'oméga- 3 sous forme de poissons ou d'huiles oméga-3, augmente ce risque.

En ce qui concerne les traitements potentiels de la maladie d'Alzheimer, la consommation d'huiles de poissons ne serait efficace qu'à titre préventif, au tout début de l'apparition des troubles, selon les résultats présentés par $\mathrm{M}$. Bryhn.

Les mécanismes impliqués rapportés par S. Cunnnane, P. Guesnet, J.M. Alessandri, F. Pifferi, T. Hartmann, T. Pillot et J. Vamecq font intervenir différents facteurs, dont l'allèle ApoE4, le potentiel anti-inflammatoire du DHA, la voie de formation des corps cétogènes et le métabolisme cérébral du glucose qui dépend: i) des transporteurs au glucose (GLUT1), ii) d'un apport adéquat en DHA (facilitateur) iii) et en ALA comme possible substrat énergétique alternatif (S. Cunnane). Dans la maladie d'Alzheimer ou l'épilepsie, les valeurs de DHA et d'utilisation du glucose sont faibles dans certaines parties du cerveau, et il existerait un « hypométabolisme ", lié à ce défaut local d'énergie.

C. Heurteaux et N. Pages ont indépendamment apporté la démonstration d'une neuroprotection induite respectivement par des injections d'ALA ou des régimes chroniques à base de colza (riches en ALA), dans des cas d'ischémie cérébrale et d'épilepsie sur modèles de souris différents.

Des méthodes non invasives d'imagerie du cerveau sont maintenant proposées pour localiser et évaluer les anomalies d'activité cérébrale dans différentes conditions expérimentales chez l'animal ou l'homme.

Ceci nous amène à la présentation de S. Rapoport qui, à cette occasion, a reçu la Médaille Chevreul. II nous a présenté ses tous derniers travaux.

D'abord, utilisant des méthodes d'imagerie (PETscan : Positron Emission Tomography) chez l'homme, il a pu mesurer la consommation d'AA et DHA par le cerveau de I'homme adulte ( 17,8 et $4,6 \mathrm{mg} / \mathrm{j}$ respectivement) et montrer que la consommation d'AA n'évolue pas avec l'âge.

Le retentissement de faibles modifications de l'apport en DHA sur les fonctions du cerveau (comportement, pathologies diverses), telles que rapportées précédemment en clinique humaine, l'a incité à étudier l'impact de conditions similaires chez l'animal, ce qui n'avait jusqu'à présent pas été fait (les études ayant porté essentiellement sur des carences sévères). D'autre part, les apports nutritionnels optimaux restent encore à définir pour le cerveau et la part que représente l'implication du foie dans la transformation de I'ALA en DHA (et du linoléique en $A A$ ) pour son utilisation dans le métabolisme cérébral n'avait jusque-là jamais été établie, ni chez l'animal, ni chez l'homme.

En quelques mots et pour résumer ce fantastique travail, il a montré que :

- Chez le rat nourri avec ce qu'il a appelé un régime suffisant (adequate diet) comportant comme seul apporten oméga-3 de ALA à 4,6 \% (colza/autre huile à 50/50), la vitesse de sécrétion hépatique du DHA, issu uniquement de la métabolisation de I'ALA circulant, est 10 fois plus élevée que la vitesse de consommation journalière du cerveau. Ainsi le foie est capable, dans ces conditions, de fournir à partir de I'ALA de source alimentaire la totalité des besoins du cerveau en DHA.

- Lorsque le régime est très peu riche en $A L A$, le foie accélère sa transformation en DHA (par une augmentation des élongases et désaturases hépatiques) et en parallèle, le renouvellement cérébral du DHA est ralenti. Cette situation conduit néanmoins à une déficience en DHA qui est alors remplacé par son "équivalent $n-6$ " (22:5n-6) et ces modifications conduisent à un défaut de fonctionnement, une plus grande vulnérabilité et une augmentation des processus inflammatoires du cerveau.

Voici donc éclaircie la situation concernant les sources de DHA du cerveau.

Pour conclure, si on sait maintenant que la nutrition joue sur la chimie du cerveau, on peut dire également qu'elle module nos «humeurs» et notre comportement, ainsi que notre capacité à développer des pathologies à tous les stades de notre vie.

On ne peut plus considérer à l'heure actuelle, comme on a pu le voir dans certains "régimes extrêmes", que les lipides soient à proscrire parce qu'ils représentent un apport calorique important, sans prendre en compte leur impact sur le cerveau, c'est-à-dire l'impact sur le comportement et la santé psychique de l'individu; sans parler du retentissement des modifications alimentaires de la mère sur l'évolution ou la maturation de l'enfant et sur la santé de l'adulte en devenir qu'il représente.

Les oméga-3 ne représentent pas un phénomène de mode comme son apparition massive dans les médias pourrait le faire penser : il s'agit seulement de remettre à sa vraie place une famille d'acides gras oubliée pendant ces soixante dernières années.

$\mathrm{Si}$ la consommation de poisson comme source de LCn-3 (EPA et DHA) est unanimement recommandée, I'alphalinolénique (ALA) contenu dans certaines huiles végétales (colza, noix...) doit retrouver son image d'acide gras indispensable, précurseur de ces LCn-3, pour une protection cérébrale et cardiaque de l'enfant à l'homme âgé.

Encore une fois, un grand merci à tous les intervenants, participants et organisateurs. II m'a été impossible de commenter ici l'ensemble des travaux présentés, mais vous trouverez tous les articles se rapportant aux présentations dans ce dossier.

Bernadette Delplanque, CR Inserm Présidente de I'AFECG

NMPA, Université Paris Sud, Orsay, France

Bernadette.Delplanque@u-psud.fr 